\title{
Thermal Joint Resistance of Conforming Rough Surfaces with Grease-Filled Interstitial Gaps
}

\author{
I. Savija, ${ }^{*}$ M. M. Yovanovich, ${ }^{\dagger}$ and J. R. Culham ${ }^{\ddagger}$ \\ University of Waterloo, Waterloo, Ontario N2L 3G1, Canada \\ and \\ E. E. Marotta ${ }^{\S}$ \\ IBM Corporation, Poughkeepsie, New York 12601
}

\begin{abstract}
Thermal joint conductance and resistance models are presented for grease-filled joints formed by conforming rough surfaces under light contact pressures. One model includes the thermal effect of contacting asperities, whereas the second, simpler model is based on conduction across the gaps only. The models are compared against recently published grease and phase-change material (PCM) data obtained at one contact pressure, copper surfaces having three levels of surface roughness, four values of grease thermal conductivity, and two values of PCM conductivity. The models and the data are found to be in agreement over a wide range of a joint parameter defined as the ratio of the effective joint roughness and the thermal conductivity of the gap substance. The models can be used to predict an upper bound on the joint conductance and a lower bound on the specific joint resistance for surfaces that are turned and milled.
\end{abstract}

\section{Nomenclature}

$\begin{array}{ll}A_{a}, A_{c}, A_{g} & =\text { apparent, contact, and gap area, } \mathrm{m}^{2} \\ c_{1} & =\text { Vickers correlation coefficient, } \mathrm{MPa} \\ c_{2} & =\text { Vickers correlation coefficient } \\ d_{V} & =\text { Vickers average diagonal, } \mu \mathrm{m} \\ H_{B} & =\text { Brinell hardness, MPa } \\ H_{B}^{*} & =\text { dimensionless Brinell hardness, } H_{B} / 3178 \\ H_{c} & =\text { contact microhardness, } \mathrm{MPa} \\ H_{V} & =\text { Vickers microhardness, } \mathrm{MPa} \\ h_{c}, h_{g}, h_{j} & =\text { contact, gap, and joint conductances, } \mathrm{W} / \mathrm{m}^{2} \cdot \mathrm{K} \\ k_{g} & =\text { grease conductivity, } \mathrm{W} / \mathrm{m} \cdot \mathrm{K} \\ k_{s} & =\text { harmonic mean thermal conductivity, } \\ & 2 k_{1} k_{2} /\left(k_{1}+k_{2}\right), \mathrm{W} / \mathrm{m} \cdot \mathrm{K} \\ k_{1}, k_{2} & =\text { solid thermal conductivities, } \mathrm{W} / \mathrm{m} \cdot \mathrm{K} \\ m & =\text { effective mean absolute asperity } \\ & \text { slope, } \sqrt{ }\left(m_{1}^{2}+m_{2}^{2}\right) \\ m_{p} & =\text { mean plane in equivalent surface } \\ m_{1}, m_{2} & =\text { mean absolute asperity slopes of surfaces } \\ m_{p_{1}}, m_{p_{2}} & =\text { mean planes in surfaces } 1 \text { and } 2 \\ P & =\text { apparent contact pressure, } \mathrm{MPa} \\ Q & =\text { joint heat transfer rate, } \mathrm{W} \\ R_{c}, R_{g}, R_{j} & =\text { contact, gap, and joint resistances, } \mathrm{K} / \mathrm{W} \\ r_{j} & =\text { specific joint resistance, } 1 / h_{j}, \mathrm{~m}^{2} \mathrm{~K} / \mathrm{W} \\ Y & =\text { separation of mean planes, } \mathrm{m} \\ \Delta T_{j} & =\text { joint temperature drop, } \mathrm{K} \\ \sigma & =\text { effective joint surface roughness, } \\ \sigma_{1}, \sigma_{2} & =\sqrt{ }\left(\sigma_{1}^{2}+\sigma_{2}^{2}\right), \mu \mathrm{m} \\ & \text { rms surface roughness, } \mu \mathrm{m} \\ & \end{array}$

Received 21 February 2002; revision received 8 November 2002; accepted for publication 19 November 2002. Copyright (C) 2003 by the authors. Published by the American Institute of Aeronautics and Astronautics, Inc., with permission. Copies of this paper may be made for personal or internal use, on condition that the copier pay the $\$ 10.00$ per-copy fee to the Copyright Clearance Center, Inc., 222 Rosewood Drive, Danvers, MA 01923; include the code 0887-8722/03 \$10.00 in correspondence with the CCC.

*M.S. Candidate, Department of Mechanical Engineering.

${ }^{\dagger}$ Distinguished Professor Emeritus, Department of Mechanical Engineering. Fellow AIAA.

*Associate Professor, Department of Mechanical Engineering, and Director, Microelectronics Heat Transfer Laboratory.

${ }^{\S}$ Senior Engineer, Development, Product Packaging, Power and Cooling Group. Senior Member AIAA.

\author{
Subscripts \\ $a, c, j=$ apparent, contact, and joint \\ $B \quad=$ Brinell \\ $s \quad=$ harmonic mean value \\ $V \quad=$ Vickers \\ $1,2=$ surfaces 1 and 2 or solids 1 and 2
}

\section{Introduction}

$\mathbf{W}$ HEN two nominally flat, rough surfaces are brought into contact by a mechanical load, a joint is created. The joint consists of numerous microcontacts formed by plastic, elastic, or elastic-plastic deformation of the highest contacting asperities and the associated gaps, as shown in Fig. 1a. There are two mean planes denoted as $m_{p_{1}}$ and $m_{p_{2}}$ that pass through each surface. The distance between the two mean planes is denoteds as $Y$, which is related to the two rms surfaces roughness, $\sigma_{1}$ and $\sigma_{2}$, through the apparent contact pressure $P$ and the microhardness $H_{c}$ of the softer contacting asperities. The actual joint can be replaced by the equivalent joint formed by a rigid, smooth plane and an equivalent nominally flat, rough surface whose effective rms roughness is $\sigma=\sqrt{ }\left(\sigma_{1}^{2}+\sigma_{2}^{2}\right)$, as shown in Fig. 1b. The mean plane denoted as $m_{p}$ lies in the rough surface, and the effective gap thickness is still $Y$, as shown in Fig. 1c.

Whenever there is steady heat transfer across a joint formed by two rough surfaces under relatively light contact pressures, a large temperature drop is observed at that joint. The temperature drop $\Delta T_{j}$ is related to the heat transfer rate $Q$ through the joint resistance $R_{j}$ or the joint conductance $h_{j}$ by the relations

$$
Q=\Delta T_{j} / R_{j}, \quad Q=h_{j} A_{a} \Delta T_{j}
$$

where $A_{a}$ is the nominal or apparent contact area. For most contact problems, the real area of contact $A_{c}$ is much smaller than the the apparent contact area, ${ }^{1}$ that is, $A_{c} / A_{a}<0.02$. Therefore, the effective gap area is approximately equal to the apparent area: $A_{g} \approx A_{a}$.

The joint conductanceand specific joint resistance, or the thermal impedance, as it is sometimes called, are related:

$$
h_{j}=1 / A_{a} R_{j} \equiv 1 / r_{j}
$$

where $r_{j}$ is introduced to represent the specific joint resistance.

It has been observed through many experiments ${ }^{1}$ that if thermal grease is introduced into the gaps formed by the contact of two flat, rough surfaces, then the thermal joint conductance $h_{j}$ is increased significantly compared to the joint conductance when the gaps are 


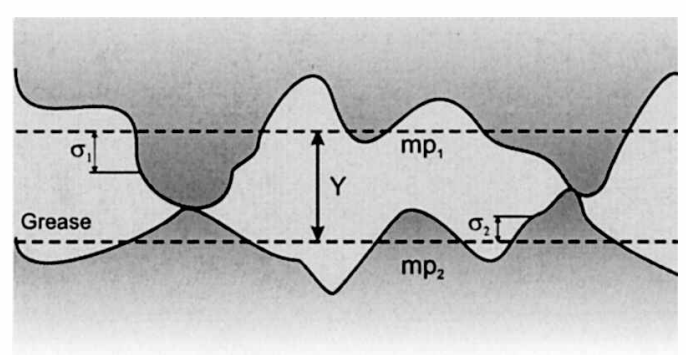

a) Two nominally flat rough surfaces

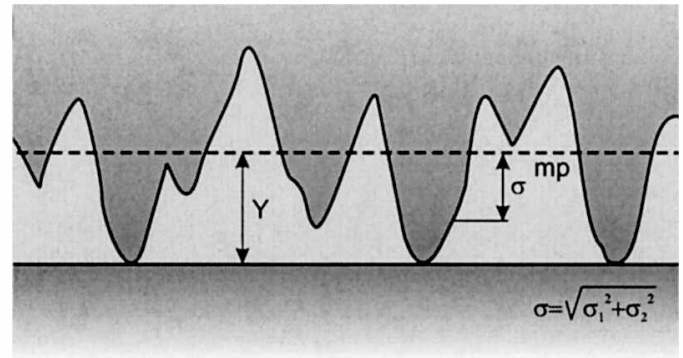

b) Equivalent rough surface, smooth plane contact

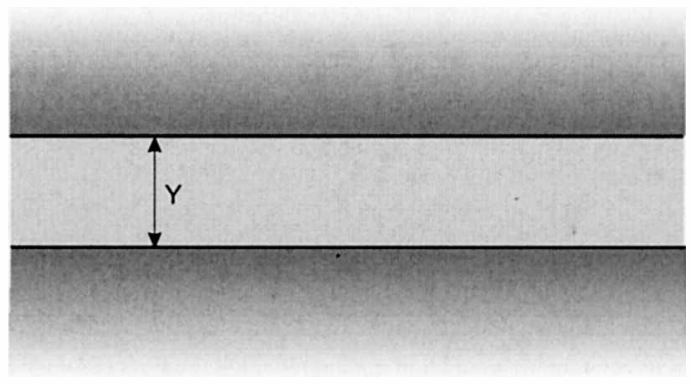

c) Equivalent uniform gap model

Fig. 1 Conforming rough surface geometries.

filled with air. It was also observed that the joint conductance has a relatively weak dependence on the contact pressure and the type of metals forming the joint and a strong dependence on the surface roughnesses $\sigma_{1}$ and $\sigma_{2}$ of the two rough surfaces and on the thermal conductivity $k_{g}$ of the grease. ${ }^{1}$

When the apparent contact pressure is very light to moderate, for example, $P_{a} / H_{c} \approx 10^{-5}-10^{-3}$, the thermal conductivities of the contacting surfaces were observed to have negligible effects on the joint conductance.

The main objective of this paper is to present models for the joint conductance and specific joint resistance for joints formed by the mechanical contact of conforming rough surfaces under light contact pressures. The gaps will be filled with some grease, which is characterized by its thermal conductivity only.

The models will give relationships between $h_{j}$ and $r_{j}$ and the joint parameters such as surface roughness, microhardness of the softer metal, and the thermal conductivity of the grease or any other substance, for example, oil or phase-change materials (PCM), that behaves like a grease.

The second objective is to compare the model predictions against the grease data presented by Cunnington, ${ }^{2}$ Getty and Tatro, ${ }^{3}$ and Seely and Chu, ${ }^{4}$ and the recently published grease and PCM data of Prasher ${ }^{5}$ and Prasher et al. ${ }^{6}$

\section{Review of Previous Work}

A review of the literature reveals that few researchers have examined experimentally the effect of thermal grease on the joint conductance or joint resistance. One of the first experimental studies was conducted by Cunnington, ${ }^{2}$ who tested two types of grease: Dow Corning 340 grease and a silicone-based vacuum grease. The joints were formed by contacting aluminum-aluminum (type 6061T4) and magnesium-magnesium (type AZ-31) solids at two contact pressures: $P=0.275$ and $0.551 \mathrm{MPa}$. The test surfaces were circu-
Table 1 Surface roughnesses and grease thermal conductivities of Prasher ${ }^{5}$

\begin{tabular}{lcc}
\hline \hline Test & $\begin{array}{c}\text { Roughness } \\
\sigma_{1}=\sigma_{2}, \mu \mathrm{m}\end{array}$ & $\begin{array}{c}\text { Conductivity } \\
k_{g}, \mathrm{~W} / \mathrm{m} \cdot \mathrm{K}\end{array}$ \\
\hline 1 & 0.12 & 3.13 \\
2 & 1.0 & 3.13 \\
3 & 3.5 & 3.13 \\
4 & 1 & 0.4 \\
5 & 3.5 & 0.4 \\
6 & 3.5 & 0.25 \\
7 & 3.5 & 0.22 \\
\hline \hline
\end{tabular}

lar rods of diameter $25.4 \mathrm{~mm}$. The aluminum surfaces were milled or turned, having surface roughness and waviness characteristics. The magnesium surfaces were milled. The thermal conductivity of DC 340 was reported as $k_{g}=0.58 \mathrm{~W} / \mathrm{m} \cdot \mathrm{K}$. That of the siliconebased grease was reported as $k_{g}=0.29 \mathrm{~W} / \mathrm{m} \cdot \mathrm{K}$. All tests were conducted under vacuum conditions. Although the test results were originally reported in tables and plots as $h_{j}$, they will be presented here as specific resistance $r_{j}$, for consistency. The measured joint resistance with DC 340 ranged from a high value of $r_{j}=0.0231$ to a low value of $0.0183 \mathrm{~m}^{2} \cdot \mathrm{K} / \mathrm{kW}$, a difference of about $26.3 \%$. Cunnington $^{2}$ conducted three sets of measurements at low contact pressure. He observed that doubling the contact pressure produced a decrease in the specific resistance of approximately $4.7 \%$ in one test, $10.3 \%$ in a second test, and $13.2 \%$ in the third test. The milled magnesium surfaces gave the lowest values of joint resistance, even though they were the roughest surfaces.

Getty and Tatro ${ }^{3}$ examined the effect of three types of greases and other interstitial substances on joint conductance. The tests were conducted with square aluminum surfaces of side dimension of approximately $75 \mathrm{~mm}$. The average surface roughness range was $0.20-7.6 \mu \mathrm{m}$. The tests were conducted at three contact pressurelevels: $P=0.0483,0.1174$, and $0.214 \mathrm{MPa}$. The interstitial substances tested were Dow Corning 340, Dow Corning silicone grease, Dow Corning silicone high-vacuum grease, silicone grease, Eccoshield SX, Eccoshield VY, and Thermoplaz. The Dow Corning grease was reported to have the highest joint resistance with a value of $r_{j}=0.0826 \mathrm{~m}^{2} \cdot \mathrm{K} / \mathrm{kW}$.

Seely and $\mathrm{Chu}^{4}$ reported one test point for DC 340 grease placed in the joint formed by copper and molybdenum surfaces having roughnesses of approximately $\sigma_{1}=\sigma_{2}=0.625 \mu \mathrm{m}$ under a contact pressure of $P=0.069 \mathrm{MPa}$. They reported a measured value of $r_{j}=0.0139 \mathrm{~m}^{2} \cdot \mathrm{K} / \mathrm{kW}$.

Recently, Prasher ${ }^{5}$ and Prasher et al. ${ }^{6}$ reported the results of an extensive experimental program to examine the effect of thermal conductivity of grease and surface roughness on the joint conductance at one contact pressure of $P=0.1 \mathrm{MPa}$. The contacting surfaces were copper. The thermal conductivities and surface roughnesses are reported in Table 1. Four types of grease were tested, having the thermal conductivity values given in Table 1 . The surface roughness of the bounding surfaces were $\sigma_{1}=\sigma_{2}=0.12,1.0$, and $3.5 \mu \mathrm{m}$. Seven values of specific joint resistance were reported. The lowest value reported was $r_{j}=0.003 \mathrm{~m}^{2} \cdot \mathrm{K} / \mathrm{kW}$, corresponding to the roughest interface and the lowest grease thermal conductivity. The highest value reported was $r_{j}=0.1 \mathrm{~m}^{2} \cdot \mathrm{K} / \mathrm{kW}$, corresponding to the smoothest interface and the highest grease thermal conductivity.

Prasher ${ }^{5}$ also reported the results of two specific joint resistance tests conducted with two PCMs having thermal conductivities of $k_{g}=0.2$ and $0.7 \mathrm{~W} / \mathrm{m} \cdot \mathrm{K}$, respectively. It is assumed that the PCM was not supported by some substrate material and that it behaved like the grease.

\section{Joint Resistance Models for Conforming Rough Surfaces}

The thermal joint resistance or conductance of a joint formed by two nominally flat, rough surfaces and that is filled with a grease, as shown in Fig. 1, depend on several geometric, physical, and thermal parameters. The resistance and conductance relations are obtained from models that are based on the following simplifying assumptions: 1) nominally flat, rough surfaces with Gaussian asperity height 
distributions; 2) random distribution of surface asperities over the apparent area; 3) load supported by the contacting asperities only; 4) light load, small nominal contact pressure, $P / H_{c} \approx 10^{-3}-10^{-5}$; 5) plastic deformation of the contacting asperities of the softer metal; and 6) homogeneous grease, completely filling the interstitial gaps, and perfectly wetting the bounding surfaces.

Two joint conductance models are considered: 1) a general joint resistance model that accounts for heat transfer through the microcontacts and the gaps and 2) a simple joint resistance model that is based on heat transfer across the gaps only.

\section{General Joint Conductance Model}

In general, the joint conductance $h_{j}$ and joint resistance $R_{j}$ depend on the contact and gap components. The joint conductance is modeled as ${ }^{1,7}$

$$
h_{j}=h_{c}+h_{g}
$$

where $h_{c}$ represents the contact conductance and $h_{g}$ the gap conductance. The joint resistance is modeled as

$$
1 / R_{j}=1 / R_{c}+1 / R_{g}
$$

The specific joint resistance is, in general, given by the relation

$$
r_{j}=1 /\left(h_{c}+h_{g}\right)
$$

The contact conductancefor conforming rough surfaces and plastic deformation of contacting asperities is ${ }^{1,7}$

$$
h_{c}=1.25 k_{s}(m / \sigma)\left(P / H_{c}\right)^{0.95}
$$

with effective joint parameters ${ }^{1,7}$

$$
k_{s}=2 k_{1} k_{2} /\left(k_{1}+k_{2}\right), \quad \sigma=\sqrt{\sigma_{1}^{2}+\sigma_{2}^{2}}, \quad m=\sqrt{m_{1}^{2}+m_{2}^{2}}
$$

If the absolute mean asperity slopes $m_{1}$ and $m_{2}$ are unknown, they can be obtained from the approximate correlation equation of Antonetti et al. ${ }^{8}$

$$
m_{i}=0.125\left(\sigma_{i} \cdot 10^{6}\right)^{0.402}, \quad i=1,2
$$

for $0.216<\sigma_{i}<9.6 \mu \mathrm{m}$.

The relative contact pressure $P / H_{c}$ is obtained from the relation proposed by Song and Yovanovich ${ }^{9}$ :

$$
P / H_{c}=\left[P / c_{1}(1.62 \sigma / m)^{c_{2}}\right]^{1 /\left(1+0.071 c_{2}\right)}
$$

where the coefficients $c_{1}$ and $c_{2}$ are obtained from the correlation equations of Vickers microhardness measurements

$$
H_{V}=c_{1}\left(d_{V} / d_{0}\right)^{c_{2}}
$$

where $c_{1}$ and $c_{2}$ are the correlation coefficients, and $d_{0}$ is some referencevalue frequently chosen, for convenience, to be $d_{0}=1 \mu \mathrm{m}$. The units of $\sigma$ in the preceding relation must be micrometers. The units of $P$ and $c_{1}$ must be consistent. The Vickers microhardness coefficients are related to $H_{B}$ for a wide range of metals. Sridhar and Yovanovich ${ }^{10}$ developed the following correlation equations:

$$
\begin{aligned}
c_{1} / 3178=\left[4.0-5.77 H_{B}^{*}+4.0\left(H_{B}^{*}\right)^{2}-0.61\left(H_{B}^{*}\right)^{3}\right] \\
c_{2}=-0.370+0.442\left(H_{B} / c_{1}\right)
\end{aligned}
$$

The correlation equations are valid in the Brinell hardness range 1300-7600 MPa.

Hegazy ${ }^{11}$ presented the following simple microhardness correlation equation for metals whose Brinell hardness ranged from 1.47$1.91 \mathrm{GPa}$ :

$$
H_{c}=\left(12.2-3.54 H_{B}\right)(\sigma / m)^{-0.26}
$$

where $H_{c}$, the effective contact hardness, and $H_{B}$ are in gigapascal, and the effective surface parameter $(\sigma / m)$ is in micrometers.

If the softer metal does not work-harden, then $H_{c} \approx H_{B}$. Because $H_{B}<H_{c}$, if we set $H_{c}=H_{B}$ in the specific joint resistancerelation, ${ }^{10}$ this will give a lower bound for the joint resistance or an upper bound for the joint conductance.

Based on the assumptions just given, the gap conductanceis modeled as an equivalent layer of thickness $Y$, shown in Fig. 1c, that is filled with grease having thermal conductivity $k_{g}$. The gap conductance is

$$
h_{g}=k_{g} / Y
$$

The gap parameter $Y$ is the distance between the mean planes passing through the two rough surfaces. This geometric parameter is related to the effective rms surface roughness $\sigma$, the contact pressure $P$, and the effective microhardness of the softer solid $H_{c}$. The mean plane separation $Y$, shown in Fig. 1c, is given by the theoretical relation

$$
Y / \sigma=\sqrt{2} \operatorname{erfc}^{-1}\left(P / H_{c}\right)
$$

for plastic deformation of the contacting asperities of the softer solid. The physical parameter $H_{c}$ represents the microhardness of the softer solid. The special function that appears is called the inverse complementary error function. Numerical methods are required to compute $Y / \sigma$ for given values of $P$ and $H_{c}$. There are two approximations for the preceding analytical relation. The first one was proposed by Yovanovich ${ }^{1}$ :

$$
Y / \sigma=1.184\left\{-\ln \left[3.132\left(P / H_{c}\right)\right]\right\}^{0.547}
$$

The second approximation, a simple power-law relation, was proposed by Antonetti ${ }^{12}$ :

$$
Y / \sigma=1.53\left(P / H_{c}\right)^{-0.097}
$$

The power-law relation shows that $Y / \sigma$ is a weak function of the relative contact pressure. The exact values computed by means of a computer algebra system, and the values calculated by means of the two approximations, are found in Table 2. The approximation of Yovanovich is more accurate over a wider range of $P / H_{c}$. For many practical applications, the ranges of the two approximations are

$$
2 \leq Y / \sigma \leq 4.75, \quad 10^{-6} \leq P / H_{c} \leq 2 \times 10^{-2}
$$

\section{Simple Joint Conductance Model}

A simple model based on gap conduction only is proposed for joints that have the following characteristics: 1) light contact pressures $(P<0.3 \mathrm{MPa}), 2)$ low-conductivity solids $(k<50 \mathrm{~W} / \mathrm{m} \cdot \mathrm{K})$, 3 ) relatively smooth surfaces $(\sigma<2.5 \mu \mathrm{m})$, and 4) high thermal conductivity grease $\left(k_{g}>1 \mathrm{~W} / \mathrm{m} \cdot \mathrm{K}\right)$. If these conditions are met, then it is assumed that $h_{c} \ll h_{g}$ and $R_{c} \gg R_{g}$. The joint conductance and joint resistance depend on the gap only; therefore,

$$
h_{j}=h_{g}, \quad 1 / R_{j}=1 / R_{g}
$$

where

$$
h_{j}=1 / A_{a} R_{j}=1 / r_{j}
$$

The simple power-law relation is recommended. By the use of this relation, the joint conductance can be expressed as

$$
h_{j}=k_{g} / \sigma(Y / \sigma)=\left[k_{g} / 1.53 \sigma\left(P / H_{c}\right)^{-0.097}\right]=1 / r_{j}
$$

Table 2 Comparisons of approximations against exact values

\begin{tabular}{lccc}
\hline \hline & \multicolumn{3}{c}{$Y / \sigma$} \\
\cline { 2 - 4 }$P / H_{c}$ & Theoretical & Yovanovich $^{1}$ & Antonetti $^{12}$ \\
\hline $10^{-6}$ & 4.753 & 4.75 & 5.84 \\
$10^{-5}$ & 4.265 & 4.25 & 4.67 \\
$10^{-4}$ & 3.719 & 3.71 & 3.73 \\
$10^{-3}$ & 3.090 & 3.09 & 2.98 \\
$10^{-2}$ & 2.326 & 2.34 & 2.39 \\
$2 \times 10^{-2}$ & 2.054 & 2.07 & 2.23 \\
\hline \hline
\end{tabular}


which clearly shows how the geometric, physical, and thermal parameters influence the joint conductance. The relation for the specific joint resistance is, therefore,

$$
r_{j}=A_{a} R_{j}=1 / h_{j}=1.53\left(\sigma / k_{g}\right)\left(P / H_{c}\right)^{-0.097}
$$

The general and simple relations for specific joint resistance will be compared against available grease and PCM test data.

\section{Comparisons of Proposed Models and Data}

The general and simple joint models are compared against recently published data for greases and PCM. ${ }^{5,6}$ The specific joint resistance data are shown plotted against the joint parameter $\sigma / k_{g}$ in Fig. 2. The grease and PCM thermal conductivities that fall in the range $0.22-3.13 \mathrm{~W} / \mathrm{m} \cdot \mathrm{K}$ are shown in the legend along with the surface roughnesses. The contacting surfaces were copper with thermal conductivity $k=397 \mathrm{~W} / \mathrm{m} \cdot \mathrm{K}$ and assumed microhardness $H_{c}=800 \mathrm{MPa}$. The apparent contact pressure was set at $P=0.1 \mathrm{MPa}$ for all tests. The data points corresponding to the roughestsurfaces and the lowest thermal conductivitiesappearin the upper right corner of Fig. 2, whereas the data points correspondingto the smoothest surfaces and the highest thermal conductivitiesappear in the lower left corner of Fig. 2. The thermal joint resistance tests ${ }^{5,6}$ were conducted with a relatively high contact microhardness, light contact pressures, and high thermal conductivity greases, resulting in an interface where the heat transfer across the joint was primarily through the gaps. For this reason, the simple gap model is used to predict the specific joint resistance. Note from the plots of the theoretical curve for $r_{j}$ and the test data that the simple joint resistance model shows, in general, the same trends as the data with respect to the joint parameter $\sigma / k_{g}$. The reported data points for the greases and the PCM lie above and below the theoretical curve. Table 3 shows the weak dependence of the specific joint resistance $10^{6} \cdot r_{j}$ on the value of the contact microhardness $H_{c}$ at $P=0.1 \mathrm{MPa}$.

Figure 3 shows the effect of the copper microcontacts on the specific joint resistance. The simple model and the general model points are shown as open squares and open triangles. When the joint parameter $\sigma / k_{g}<4$, there are very small differences between the two model predictions. However, when $\sigma / k_{g}>5$, the differences become much larger. This shows that if the thermal conductivity of the contacting asperities is high, for example, $k_{s}=397 \mathrm{~W} / \mathrm{m} \cdot \mathrm{K}$, and the thermal conductivity of the grease is low, for example, $k_{g}=0.22 \mathrm{~W} / \mathrm{m} \cdot \mathrm{K}$, then the general model should be used.

Figure 4 shows the comparison between the model predictions and the data of Cunnington, ${ }^{2}$ Getty and Tatro, ${ }^{3}$ and Seely and Chu. ${ }^{4}$

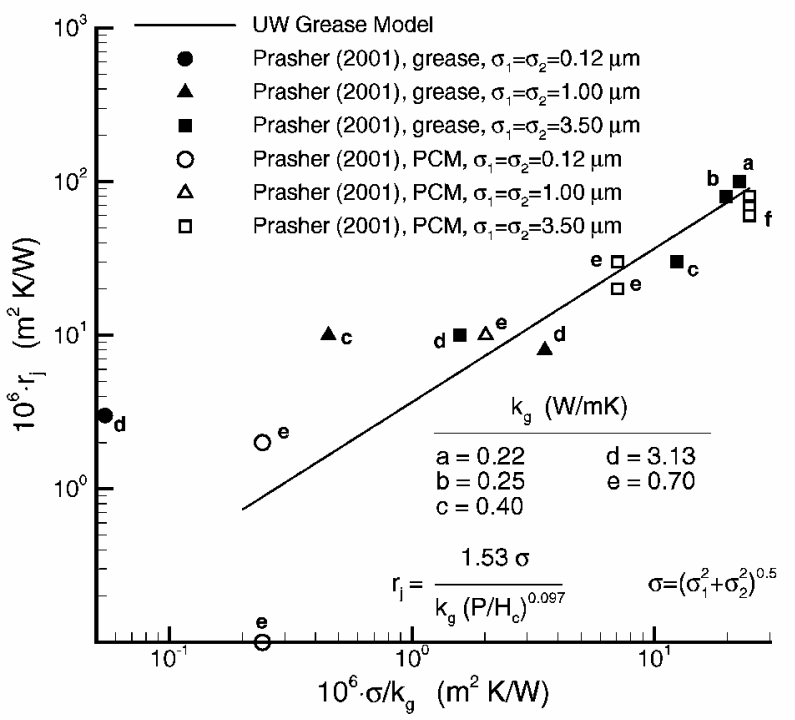

Fig. 2 Comparison of grease and phase change models vs published data.
Table 3 Sensitivity of simple joint model to $H_{c}$

\begin{tabular}{|c|c|c|c|}
\hline Test & $H_{c}=600$ & $H_{c}=800$ & $H_{c}=1000$ \\
\hline 1 & 0.193 & 0.199 & 0.203 \\
\hline 2 & 1.610 & 1.655 & 1.691 \\
\hline 3 & 5.636 & 5.794 & 5.921 \\
\hline 4 & 12.58 & 12.93 & 13.21 \\
\hline 5 & 44.05 & 45.29 & 46.28 \\
\hline 6 & 70.45 & 72.43 & 74.01 \\
\hline 7 & 80.06 & 82.31 & 84.11 \\
\hline
\end{tabular}

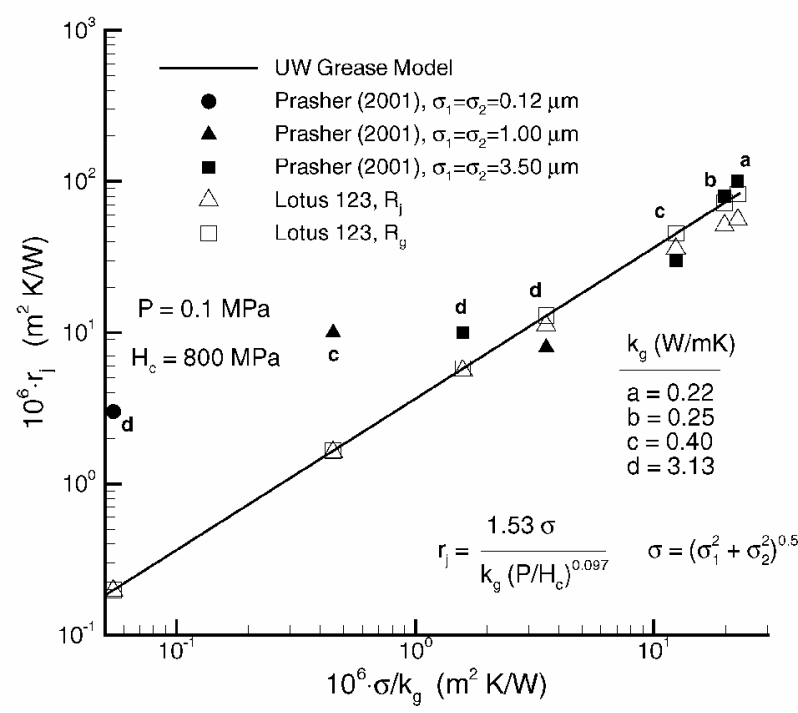

Fig. 3 Effect of copper microcontacts on specific joint resistance.

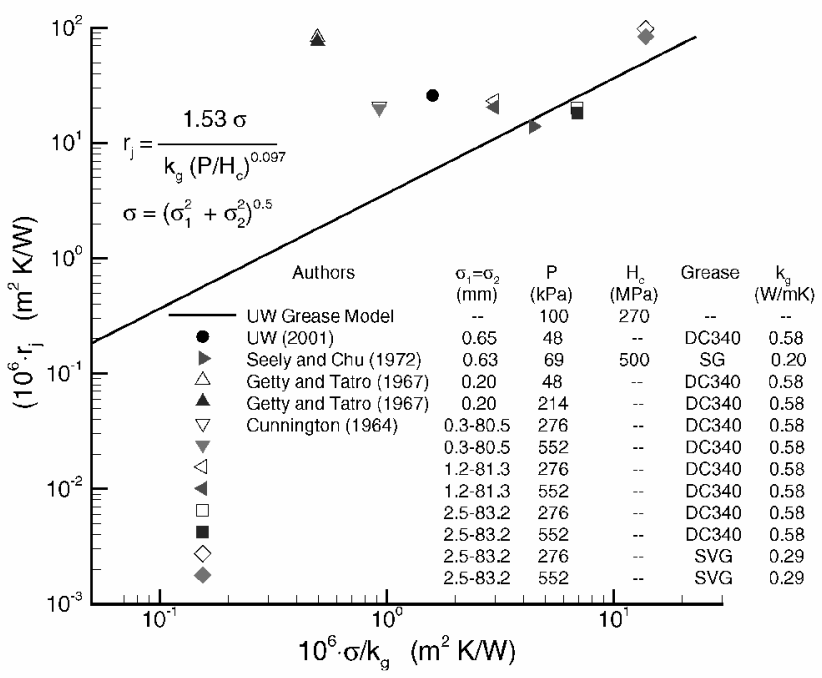

Fig. 4 Model validation.

The parameters of each test are shown in the legend. The surfaces were turned and milled. The surface roughnesses were reported to lie in a wide range of values. The joint parameter $\sigma / k_{g}$ was based on the average value of the surface roughness range and the reported values of $k_{g}$. The data clearly show a weak dependence of $r_{j}$ on $P$. The data for $\sigma / k_{g}<3$ are significantly above the simple model predictions. The large difference may be the result of several factors, such as the extremely rough surface profile associated with turned and milled surfaces, as well as the effects of surface waviness. The one exception is the single point from Seely and $\mathrm{Chu}^{4}$ that lies very close to the theoretical curve.

The sensitivity of the simple specific joint resistance model to the microhardness is shown Table 3 for the seven grease test points of Prasher. ${ }^{5}$ The units of $H_{c}$ in Table 3 are megapascal. It can be seen that a large variationin $H_{c}$ produces a small change in the value of $r_{j}$. 


\section{Summary}

Models have been proposed to predict joint conductances and specific joint resistances for conforming rough surfaces whose contacting asperities undergo plastic deformation. The general, more complex model can be used for a wide range of contact pressures where heat transfer across the joints occurs through the microcontacts and the gaps that are filled with grease or other substances that behave like a grease. A simple model based on conduction through the gaps only was proposed for joints formed by low thermal conductivity solids, high thermal conductivity greases, and relatively smooth surfaces at light to moderate contact pressures.

The proposed models, when compared against recently published test data for four greases and two PCM substances, showed trends similar to the test data with respect to a joint parameter that is based on the ratio of the effective joint roughness to the thermal conductivity of the gap substance.

There was nominal agreement between the model predictions and the data reported for milled and turned surfaces that had large variations in the surface roughnesses. The largest differences between the test data and the model predictions occurred at low values of the joint parameter. The observed differences may be partially due to the measured nonflatness in the surfaces used in the experiments.

Because the proposed models are based on nominally flat, rough surfaces, they can be used to predict upper bounds on the joint conductances and lower bounds on the specific joint resistances of nonflat, milled, turned, or ground surfaces.

Further grease and PCM tests are required to validate the proposed models over a range of contact pressures.

\section{Acknowledgments}

The authors acknowledge the continued financial support of the Natural Science and Engineering Research Council of Canada and the Centre for Microelectronics Assembly and Packaging.

\section{References}

${ }^{1}$ Yovanovich, M. M., "New Contact and Conductance Correlations for Conforming Rough Surfaces," Spacecraft Radiative Transfer and Temperature Control, Vol. 83, Progress in Astronautics and Aeronautics, AIAA, New York, 1982, pp. 83-95.

${ }^{2}$ Cunnington, G. R., Jr., "Thermal Conductance of Filled Aluminum and Magnesium Joints in a Vacuum Environment,' American Society of Mechanical Engineers, ASME Paper 64-WA/HT-40, Nov./Dec. 1964.

${ }^{3}$ Getty, R. C., and Tatro, R. E., "Spacecraft Thermal Joint Conduction," AIAA Paper 67-316, April 1967.

${ }^{4}$ Seely, J. H., and Chu, R. C., Heat Transfer in Microelectronic Equipment, A Practical Guide, Marcel Dekker, New York, 1972, pp. 40-46.

${ }^{5}$ Prasher, R. S., "Surface Chemistry and Characteristics Based Model for the Thermal Contact Resistance of Fluidic Interstitial Thermal Interface Materials," Journal of Heat Transfer, Vol. 123, No. 5, 2001, pp. 969-975.

${ }^{6}$ Prasher, R. S., Simmons, C., and Solbrekken, G., "Thermal Contact Resistance of Phase Change and Grease Type Polymeric Materials," Proceedings of the International Mechanical Engineering Congress and Exposition, MED-Vol. 11, American Society of Mechanical Engineers, Fairfield, NJ 2000, pp. 461-466.

${ }^{7}$ Yovanovich, M. M., Culham, J. R., and Teertstra, P., "Calculating Interface Resistance," Electronics Cooling, Vol. 3, No. 2, 1997, pp. 1-7; also http://www.electronics-cooling.com/Resources/EC_Articles/MAY97/ article3.htm.

${ }^{8}$ Antonetti, V. W., Whittle, T. D., and Simons, R. E., “An Approximate Thermal Contact Conductance Correlation," Experimental/Numerical Heat Transfer in Combustion and Phase Change, HTD-Vol. 170, American Society of Mechanical Engineers, Fairfield, NJ, 1991, pp. 35-42.

${ }^{9}$ Song, S., and Yovanovich, M. M., "Relative Contact Pressure: Dependence on Surface Roughness and Vickers Microhardness," Journal of Thermophysics and Heat Transfer, Vol. 2, No. 1, 1988, pp. 43-47.

${ }^{10}$ Sridhar, M. R., and Yovanovich, M. M., "Empirical Methods to Predict Vickers Microhardness,” WEAR, Vol. 193, No. 1, 1996, pp. 91-98.

${ }^{11}$ Hegazy, A. A., "Thermal Joint Conductance of Conforming Rough Surfaces: Effect of Surface Micro-Hardness Variation,” Ph.D. Dissertation, Dept. of Mechanical Engineering, Univ. of Waterloo, Waterloo, ON, Canada, 1985.

${ }^{12}$ Antonetti, V. W., "On the Use of Metallic Coatings to Enhance Thermal Contact Conductance," Ph.D. Dissertation, Dept. of Mechanical Engineering, Univ. of Waterloo, Waterloo, ON, Canada, 1983. 